\title{
Performance of a 97-element ALPAO membrane magnetic deformable mirror in an adaptive optics - optical coherence tomography system for in vivo imaging of the human retina
}

\author{
Robert J. Zawadzki, ${ }^{* 1}$ Steven M. Jones, ${ }^{2}$ Sandra E. Balderas-Mata, ${ }^{1,3}$ Sylwia M. Maliszewska, ${ }^{1,4}$ \\ Scot S. Olivier, ${ }^{2}$ and John S. Werner ${ }^{1}$ \\ ${ }^{1}$ Vision Science and Advanced Retinal Imaging Laboratory (VSRI) and Dept. of Ophthalmology \& Vision Science, \\ University of California Davis, 4860 Y Street, Ste. 2400, Sacramento, CA 95817, USA \\ ${ }^{2}$ Lawrence Livermore National Laboratory, 6000 East Avenue, Livermore, CA 94550, USA \\ ${ }^{3}$ National Institute for Astrophysics, Optics \& Electronics (INAOE), A.P. 51 y 216, Puebla, 72000, Mexico \\ ${ }^{4}$ Institute of Physics, Nicolaus Copernicus University, ul. Grudziadzka 5/7, PL-87-100 Torun, Poland
}

Received November 22, 2011; accepted December 18, 2011; published December 31, 2011

\begin{abstract}
We present an evaluation of two different configurations of the ALPAO 97-actuator membrane magnetic deformable mirror for wavefront correction, using either 7 or 9 actuators across the eye pupil. These tests included monitoring of aberration correction for a human subject. This AO-sub system is part of the UC Davis high-resolution human retinal imaging adaptive optics - optical coherence tomography (AO-OCT) instrument. AO-OCT allows the three-dimensional (3D) visualization of different retinal structures in vivo with high volumetric resolution $\left(3 \times 3 \times 3 \mu \mathrm{m}^{3}\right)$.
\end{abstract}

The combination of adaptive optics (AO) with any retinal imaging technique allows for improved lateral, and in the case of the confocal scanning laser ophthalmoscope (cSLO) and fundus camera, also axial resolution. The AOOCT instrument at UC Davis has been under development for several years, and has demonstrated the utility of this technology for microscopic, volumetric, in vivo retinal imaging [1]-[3]. In our adaptive optics system a Hartmann-Shack wavefront sensor and a deformable mirror operate in a closed loop. This approach, to improve the transverse resolution for retinal imaging by correction of the eye's static and dynamic aberrations, has been demonstrated first in standard AO-fundus cameras [4] followed by AO-SLO [5]. The latter modality allows realtime observation of microscopic retinal structures in human eyes, including the photoreceptor cone mosaic, nerve fiber layer and microscopic blood flow in capillaries. Due to the limited axial resolution of these systems, however, they cannot be used to image other retinal layers or to create detailed 3D reconstructions of retinal volumes, a key advantage associated with OCT imaging [6]-[8].

As previously reported, the initial configuration of the UC Davis AO sub-system used a 35-actuator AOptix bimorph deformable mirror (DM) for low-order, high-

*E-mail: rjzawadzki@ucdavis.edu stroke correction [9] and a 140-actuator Boston Micromachines MEMS DM for high-order correction. The performance of the AO-subsystem of this instrument was previously evaluated and the results were presented by Evans et al. [10]. Later we replaced this configuration with a single novel membrane magnetic deformable mirror with increased stroke and actuator count. Initially, we implemented a configuration that used only the center $10.5 \mathrm{~mm}$ diameter of the DM for wavefront correction, equivalent to 69-actuators of the ALPAO membrane magnetic deformable mirror [3] (7 actuators across the eye pupil). In that configuration both the AOptix and MEMS DM's were removed from the system. A flat mirror was placed at the MEMS DM position and the ALPAO DM was placed at the AOptix position. Figure 1 shows the schematic of our AO system using two vs. one deformable mirror.
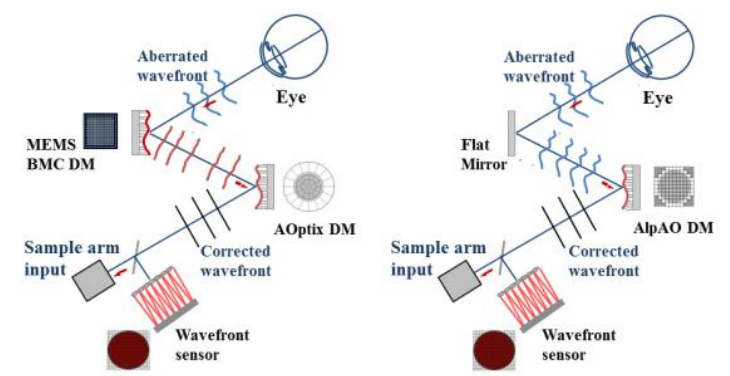

Fig. 1. Schematic of the AO system. Left: AO system with 2 deformable mirrors; Right: AO system with one Deformable mirror.

Note that all the other components remained the same.

This simple swap of the deformable mirrors, without changes in any other optical components, as shown in Fig. 1 , resulted in non-optimal use of that DM. Recently, we have upgraded elements of our sample arm optics to accommodate a larger diameter of the ALPAO DM (13.5 $\mathrm{mm})$ and to allow utilization of the whole reflecting 
surface ( 9 actuators across eye pupil). Figure 2 shows images of a subject's pupil scaled to the actuator geometry for the two configurations of DM used in our AO system.
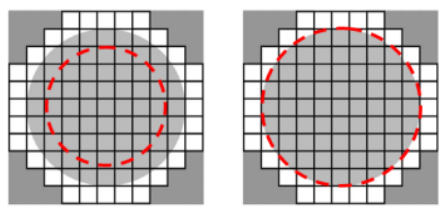

Fig. 2. Comparison of the eye pupil size imaged to the ALPAO DM surface for two configurations tested in this paper. The grey area represents the mirror surface. Left: configuration using 69-elements of the ALPAO DM; Right: configuration using 97-elements of the ALPAO DM. The mirror size conjugate to the subject's eye pupil (diameter 6.75 $\mathrm{mm}$ ) is marked by the red dashed circle on each DM and equals 10.5 $\mathrm{mm}$ and $13.5 \mathrm{~mm}$, respectively.

The AO-OCT system used for the experiments presented in this paper occupies $1.5 \times 2 \mathrm{~m}$ of a standard laboratory optical table. The sample arm utilizes a Hartmann-Shack wavefront sensor and a single 97actuator ALPAO membrane magnetic deformable mirror. The same light source is used for both OCT and Hartmann-Shack wavefront sensing, allowing simultaneous operation of AO and OCT. Two independent $\mathrm{PCs}$ are used, one for the AO control subsystem and one for the OCT sub system. A bite-bar and forehead-rest assembly was mounted on a motorized $\mathrm{X}-\mathrm{Y}-\mathrm{Z}$ translation stage to permit precise positioning of the subject's eye. Our Hartmann-Shack wavefront sensor uses a 20x20 lenslet array with $500 \mu \mathrm{m}$ pitch and $30 \mathrm{~mm}$ focal length. The 284 lenslets from the array were used to sample the wavefront at an $\sim 18 \mathrm{~Hz}$ frame rate synchronized to the OCT system frame rate. Our AO control software allows the focus of the AO-OCT beam to be placed onto any retinal structures of interest while still correcting higher-order aberrations. The system operator determines the axial layer of interest by adding a preset defocus to the WFS centroids reference file. An image from the wavefront sensor is used to monitor the position of the eye's pupil while fixation is directed to an external target to minimize head and eye motion and to allow precise imaging of different retinal eccentricities. To ensure the maximum pupil size and minimize fluctuations in accommodation, the subject's eye is dilated and cyclopleged with $2.5 \%$ Phenylephrine and $1 \%$ Tropicamide. To test the system performance we monitored the AO-system during wavefront measurement and correction for the human eye in vivo. These experiments confirmed the system's ability to correct lowand high-order aberrations. The next two figures summarize the performance of the AO system in the first configuration with 7 actuators across the pupil
Figure 2 shows an example of the total reconstructed wavefront RMS error before and during AO correction as well as a $3 \mathrm{D}$ representation of the wavefront.
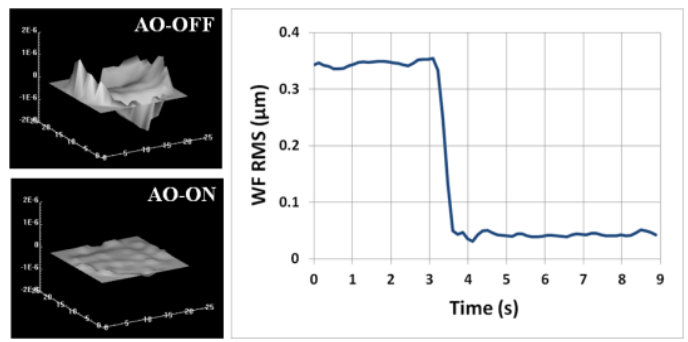

Fig. 3. The ocular wavefront measured by the $\mathrm{H}-\mathrm{S}$ wavefront sensor before and during AO-correction for the first DM configuration. Left plots show $3 \mathrm{D}$ representation of the wavefront map before (top) and during (bottom) wavefront correction. The right plot shows the trace of the total wavefront RMS error over time.

Figure 4 shows an example trace and a 3D visualization of the reconstructed point-spread function (PSF) before and during AO correction for the same DM configuration (with 7 actuators across the pupil), for the data shown in Figure 3.
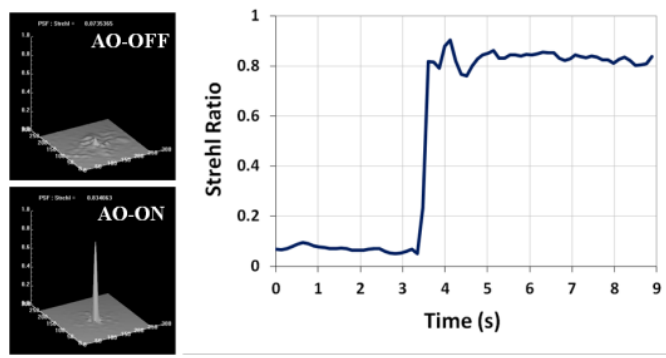

Fig. 4. Point-spread function (PSF) and calculated Strehl Ratio (SR) before and during AO-correction for the first AO configuration. Left plots show 3D representation of the PSF before (top) and during (bottom) wavefront correction. Right plot show trace of the SR.

The next two figures summarize the performance of the AO system in the second configuration, with 9 actuators across the pupil. Figure 5 shows the total reconstructed wavefront RMS error before and during AO correction as well as a $3 \mathrm{D}$ representation of the wavefront acquired from the same subject presented in Figures 3 and 4.
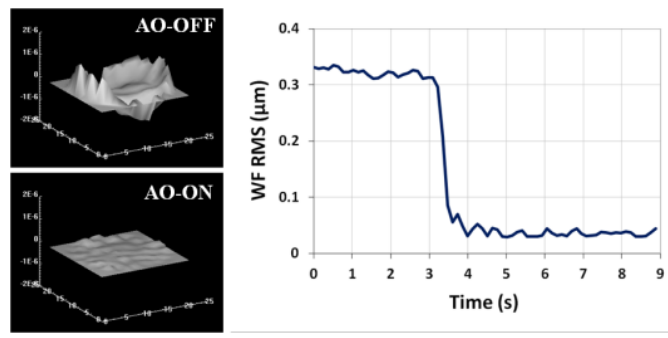

Fig. 5. The ocular wavefront measured by the $\mathrm{H}-\mathrm{S}$ wavefront sensor before and during AO-correction for the second AO configuration. Other details as in Figure 3. 
Figure 6 shows example traces and 3D visualization of the reconstructed PSF before and during AO correction for the same DM configuration (with 9 actuators across pupil) using the data shown in Figure 5.
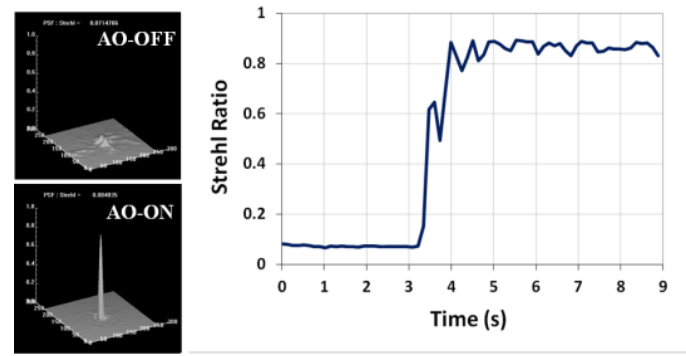

Fig. 6. Point-spread function (PSF) and calculated SR before and during AO-correction for the second AO configuration. Left plots show

$3 \mathrm{D}$ representation of the PSF before (top) and during (bottom) wavefront correction. The right plot shows the trace of the SR over time.

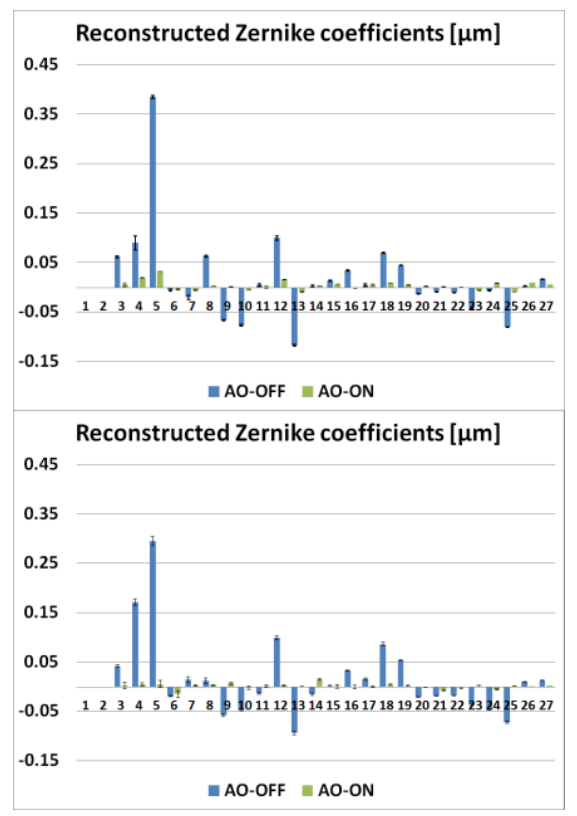

Fig. 7. Reconstructed averaged Zernike coefficients measured before (blue) and during (green) AO correction for DM configurations with 7 (top) and 9 actuators across the pupil (bottom). Numbers correspond to the following Zernike coefficients: second-order aberrations: 3,5 astigmatism, 4 - defocus; third-order aberrations: 6,9 - trefoil, 7,8 -

coma; fourth- order aberrations: $10,14=$ tetrafoil, $11,13-2$ nd astigmatism, 12 - spherical aberrations; fifth-order aberrations 15-20; sixth-order aberrations 21-27;

As can be seen, both configurations of the AO system can significantly compensate for aberrations and maintain a low RMS wavefront error during its closed-loop operation. However, as expected, the configuration with 9 actuators across the eye pupil achieved better wavefront correction. The ability to correct the wavefront error in real time, while imaging, allows compensation of the eye's temporal aberration fluctuations. To better estimate the performance of both configurations, the Zernike representation of the reconstructed wavefront was calculated and analyzed.

Figure 7 shows the Zernike coefficients associated with the wavefront presented for both configurations in Figures 3 and 5.

Note that a clear reduction in the low-order and highorder aberration modes was observed. Adaptive optics using a single deformable mirror with increased stroke and actuator count offers a good compromise if compared with our two DM (woofer-tweeter) configuration. It allows for more compact optical design and simplifies AO control software. The performance of the novel membrane magnetic deformable mirror should be sufficient to correct aberrations of most subjects to successfully achieve cellular resolution retinal imaging [11]-[12].

We gratefully acknowledge the contributions of current and former VSRI laboratory members. This research was supported by the National Eye Institute (grant EY014743) and Research to Prevent Blindness.

\section{References}

[1] R.J. Zawadzki, S. Jones, S. Olivier, M. Zhao, B. Bower, J. Izatt, S Choi, S. Laut, and J. Werner, Opt. Exp. 13, 8532 (2005), http://www.opticsinfobase.org/oe/abstract.cfm?URI=oe-13-21-8532

[2] R.J. Zawadzki, B. Cense, Y. Zhang, S.S. Choi, D.T. Miller, and J.S. Werner, Opt. Exp. 16, 8126 (2008), http://www.opticsinfobase.org/oe/abstract.cfm?URI=oe-16-11$\underline{8126}$

[3] R.J. Zawadzki, S.M. Jones, S. Pilli, S. Balderas-Mata, D. Kim, S.S. Olivier, and J.S. Werner, Biomed. Opt. Exp. 2, 1674 (2011), http://www.opticsinfobase.org/boe/abstract.cfm?URI=boe-2-6$\underline{1674}$

[4] J. Liang, D.R. Williams and D.T. Miller, J. Opt. Soc. Am. A 14, 2884 (1997), http://www.opticsinfobase.org/josaa/abstract.cfm?URI=josaa-14$\underline{11-2884}$

[5] A. Roorda, F. Romero-Borja, W.J. Donnelly III, H. Queener, T.J. Hebert, and M.C.W. Campbell, Opt. Exp. 10, 405 (2002), http://www.opticsexpress.org/abstract.cfm?URI=OPEX-10-9-405

[6] D. Huang, E. A. Swanson, C.P. Lin, J.S. Schuman, W.G. Stinson, W. Chang, M.R. Flotte, K. Gregory, C.A. Puliafito, Science 254, 1178 (1991).

[7] W. Drexler and J.G. Fujimoto, J. Biomed. Opt. 12, 041201 (2007).

[8] M. Wojtkowski, Applied Optics 49, D30 (2010), http://www.opticsinfobase.org/ao/abstract.cfm?URI=ao-49-16$\underline{\mathrm{D} 30}$

[9] D.A. Horsley, H. Park, S.P. Laut and J.S. Werner, Sensors and Actuators A: Physical 134, 221 (2007).

[10] J.W. Evans, R.J. Zawadzki, S.M. Jones, S.S. Olivier, and J.S. Werner, Opt. Exp. 17, 13768 (2009), http://www.opticsinfobase.org/oe/abstract.cfm?URI=oe-17-1613768

[11] N. Doble, D.T. Miller, G. Yoon, and D.R. Williams, Appl. Opt. 46, 4501 (2007), http://www.opticsinfobase.org/ao/abstract.cfm?URI=ao-46-20$\underline{4501}$

[12] N. Devaney, E. Dalimier, T. Farrell, D. Coburn, R. Mackey, D. Mackey, F. Laurent, E. Daly, and C. Dainty, Appl. Opt. 47, 6550 (2008), http://www.opticsinfobase.org/ao/abstract.cfm?URI=ao$\underline{47-35-6550}$ 